\title{
Regularity and nonexistence results for some free-interface problems related to Ginzburg-Landau vortices
}

\author{
NAM Q. LE \\ Courant Institute of Mathematical Sciences, 251 Mercer St, New York, NY 10012, USA \\ E-mail:quangle@cims.nyu.edu
}

[Received 10 October 2007 and in revised form 17 July 2008]

\begin{abstract}
We study regularity and nonexistence properties for some free-interface problems arising in the study of limiting vorticities associated to the Ginzburg-Landau equations with magnetic field in two dimensions. Our results imply in particular that if these limiting vorticities concentrate on a smooth closed curve then they have a distinguished sign; moreover, if the domain is thin then solutions of the Ginzburg-Landau equations cannot have a number of vortices much larger than the applied magnetic field.
\end{abstract}

\section{Introduction and main results}

\subsection{Presentation of the problem}

In this paper, we are interested in the equation

$$
\operatorname{div} T_{\mu}=0 \quad \text { in } \Omega
$$

where $T_{\mu} \equiv\left(\left(T_{\mu}\right)_{i j}\right)_{1 \leqslant i, j \leqslant 2}$ defined by

$$
T_{\mu}=\left(\begin{array}{cc}
\frac{1}{2}\left(\left(\partial_{2} h_{\mu}\right)^{2}-\left(\partial_{1} h_{\mu}\right)^{2}+h_{\mu}^{2}\right) & -\partial_{1} h_{\mu} \partial_{2} h_{\mu} \\
-\partial_{1} h_{\mu} \partial_{2} h_{\mu} & \frac{1}{2}\left(\left(\partial_{1} h_{\mu}\right)^{2}-\left(\partial_{2} h_{\mu}\right)^{2}+h_{\mu}^{2}\right)
\end{array}\right)
$$

is the symmetric stress-energy tensor associated to the solution $h_{\mu} \in H^{1}(\Omega)$ of the equation

$$
\begin{cases}-\Delta h+h=\mu & \text { in } \Omega \\ h=c & \text { on } \partial \Omega(c \equiv 0 \text { or } c \equiv 1) .\end{cases}
$$

Equation 1.1, understood in the sense of distributions as $\partial_{1}\left(T_{\mu}\right)_{i 1}+\partial_{2}\left(T_{\mu}\right)_{i 2}=0$ for $i=1,2$, arises in the study of limiting vorticities for the Ginzburg-Landau equations in superconductivity which we will discuss in the next subsection. In the context of Ginzburg-Landau theory, (1.2) corresponds to the limit of the "London equation", and (1.1), obtained by passing to the limit in the stress-energy tensor associated to the Ginzburg-Landau energy $G_{\varepsilon}$ (see $(1.4)$ ), is a criticality condition on the limiting measures $\mu$ of critical points of $G_{\varepsilon}$. In $\sqrt{1.1}-(1.2)$, the domain $\Omega$ is a smooth bounded domain in $\mathbb{R}^{2}$ corresponding to a section of the superconducting material and $\mu$ is a measure belonging to $H^{-1}(\Omega)$, the dual of the Sobolev space $H_{0}^{1}(\Omega)$. Finally, we note that $(1.1)$ can be formally written as $\nabla h_{\mu} \mu=0$ and thus $\mu$ is a (weak version of) stationary solution of the evolution problem

$$
\frac{\mathrm{d}}{\mathrm{d} t} \mu(t)-\operatorname{div}\left(\nabla h_{\mu(t)}|\mu(t)|\right)=0 \quad \text { in } \Omega
$$


which has recently been studied using a gradient flow approach by Ambrosio and Serfaty. See [1] and the references therein for previous studies of (1.3).

If $\mu \in L^{p}(\Omega)$ for some $p>1$, then it was proved in [11, Theorem 13.1] that for $c \equiv 1$, the solution $h_{\mu} \in H^{1}(\Omega)$ to $11.1-1.2$ satisfies $0 \leqslant h_{\mu} \leqslant 1$ and $\mu$ is in fact a nonnegative $L^{\infty}$ function and that for $c \equiv 0,(1.1)-(1.2)$ has no solutions except for the trivial measure $\mu=0$.

In the present paper, we investigate equation (1.1) with measures $\mu$ more singular than those considered above: $\mu$ is only in $H^{-1}(\Omega)$. A typical example of $\mu \in H^{-1}(\Omega)$ is the arclength measure along a smooth closed curve in $\Omega$ with some weight. It is precisely this case that we will consider in the paper. At first, this setting seems to be restrictive; however, when $\mu$ is viewed as the limiting vorticity for the Ginzburg-Landau equations, this situation is quite natural as can be seen in a recent result of Aydi [2]. Thus, in the following, we consider measures $\mu \in H^{-1}(\Omega)$ having support supp $\mu=\Sigma$, a smooth closed curve in $\Omega$ and being absolutely continuous with respect to the arclength measure on $\Sigma$ with nowhere-zero density.

We will be in particular interested in the existence and nonexistence results for (1.1)-(1.2) depending on $\mu$, the regularity of $T_{\mu}$ and $h_{\mu}$, the sign of $\mu$ and its positivity depending on the spectral properties of the domain $\Omega$. These problems in the end have some similar flavor to the studies of free boundaries and their regularity (see also Section 4 ; ; however, we have not seen many results on similar questions.

\subsection{Connections to Ginzburg-Landau vortices}

Our study of the equation (1.1) is motivated by two open problems (Problems $17 \& 18$ ) in the book of Sandier and Serfaty [11] about the limiting vorticities for the critical points $\left\{\left(u_{\varepsilon}, A_{\varepsilon}\right)\right\}_{\varepsilon>0}$ of the Ginzburg-Landau energy in superconductivity

$$
G_{\varepsilon}(u, A)=\frac{1}{2} \int_{\Omega}\left(|\nabla u-i A u|^{2}+\frac{1}{2 \varepsilon^{2}}\left(1-|u|^{2}\right)^{2}+\left|h-h_{\mathrm{ex}}\right|^{2}\right) .
$$

Here $u$ is a complex-valued function called the order parameter and its isolated zeros are called vortices; $h_{\mathrm{ex}}>0$ is the intensity of the applied magnetic field; $A: \Omega \rightarrow \mathbb{R}^{2}$ is the vector potential and $h=\operatorname{curl} A=-\partial_{2} A_{1}+\partial_{1} A_{2}$ is the induced magnetic field.

There has been much interest in the limiting vorticity measures associated to critical points of the Ginzburg-Landau energy; see the books by Bethuel, Brezis and Hélein [3] and Sandier and Serfaty [11] and the references therein for an abundant literature on the subject.

In the following, we summarize some results and recall some open problems concerning Ginzburg-Landau vortices in [11] (see also [10]) that are closely related to [1.1]-(1.2].

Let $\left\{\left(u_{\varepsilon}, A_{\varepsilon}\right)\right\}_{\varepsilon>0}$ be critical points of $G_{\varepsilon}$. Then

$$
-\nabla^{\perp} h_{\varepsilon}=\left(i u_{\varepsilon}, \nabla u_{\varepsilon}-i A_{\varepsilon} u_{\varepsilon}\right) \quad \text { in } \Omega, \quad h_{\varepsilon}=h_{\mathrm{ex}} \quad \text { on } \partial \Omega .
$$

Here $\nabla^{\perp}$ denotes the operator $\left(-\partial_{2}, \partial_{1}\right)$ and $(\cdot, \cdot)$ the scalar product in $\mathbb{C}$ identified with $\mathbb{R}^{2}$, i.e., $(a, b)=(\bar{a} b+a \bar{b}) / 2$. The important quantity carrying the topological information on the vortices of $u_{\varepsilon}$ is the vorticity $\mu_{\varepsilon}$ defined by

$$
\mu_{\varepsilon}=\mu_{\varepsilon}\left(u_{\varepsilon}, A_{\varepsilon}\right)=\operatorname{curl}\left(i u_{\varepsilon}, \nabla u_{\varepsilon}-i A_{\varepsilon} u_{\varepsilon}\right)+\operatorname{curl} A_{\varepsilon} .
$$

Taking the curl of $\left(1.5\right.$, one obtains $-\Delta h_{\varepsilon}+h_{\varepsilon}=\mu_{\varepsilon}$ with $\mu_{\varepsilon}$ approximately $2 \pi \sum_{i} d_{i}^{\varepsilon} \delta_{a_{i}^{\varepsilon}}$ (see (1.6). This is what is called the London equation in physics. The $a_{i}^{\varepsilon}$ 's are essentially the vortices with degrees $d_{i}^{\varepsilon}$. 
Now, we can recall the following

THEOREM 1.1 ([11, Theorem 13.1]) Let $\left\{\left(u_{\varepsilon}, A_{\varepsilon}\right)\right\}_{\varepsilon>0}$ be critical points of $G_{\varepsilon}$ that satisfy the energy bound $G_{\varepsilon}\left(u_{\varepsilon}, A_{\varepsilon}\right) \leqslant C_{0} \varepsilon^{\alpha-1}$ for any $\varepsilon>0$ where $\alpha>2 / 3$ is independent of $\varepsilon$. Then the vorticity $\mu_{\varepsilon}$ can be approximated by a sum of Dirac masses in the sense that for any $\varepsilon>0$ there exists a measure $v_{\varepsilon}$ of the form $2 \pi \sum_{i} d_{i}^{\varepsilon} \delta_{a_{i}^{\varepsilon}}$, where the sum is finite, $a_{i}^{\varepsilon} \in \Omega$ and $d_{i}^{\varepsilon} \in \mathbb{Z}$ for every $i$, such that

$$
\left\|\mu_{\varepsilon}-v_{\varepsilon}\right\|_{W^{-1, p}(\Omega)}\left\|\mu_{\varepsilon}-v_{\varepsilon}\right\|_{C^{0}(\Omega)^{*}} \rightarrow 0
$$

for some $p \in(1,2)$.

Let $\left\{v_{\varepsilon}\right\}_{\varepsilon>0}$ be any measure of the form $2 \pi \sum_{i} d_{i}^{\varepsilon} \delta_{a_{i}^{\varepsilon}}$ satisfying $(1.6)$ and set $n_{\varepsilon}=\sum_{i}\left|d_{i}^{\varepsilon}\right|$, the total degree of the vortices. There are four regimes to distinguish according to the ratio of $n_{\varepsilon}$ to the external field $h_{\text {ex }}$ : possibly after passing to a subsequence, one of the following holds:

(0) $n_{\varepsilon}=0$ for any $\varepsilon$ small enough and then $\mu_{\varepsilon}$ tends to 0 in $W^{-1, p}(\Omega)$.

(1) $n_{\varepsilon}=o\left(h_{\mathrm{ex}}\right)$ is nonzero for $\varepsilon$ small enough, and then $\mu_{\varepsilon} / n_{\varepsilon}$ converges in $W^{-1, p}(\Omega)$ to a measure $\mu$ such that $\mu \nabla H_{0}=0$. Hence the support of $\mu$ is contained in the set of critical points of $H_{0}$, the solution to the London equation, i.e., $[1.2)$ with $\mu \equiv 0$ and $c \equiv 1$.

(2) $h_{\mathrm{ex}} \sim \lambda n_{\varepsilon}$ with $\lambda>0$ and then $\mu_{\varepsilon} / h_{\mathrm{ex}}$ converges in $W^{-1, p}(\Omega)$ to a measure $\mu$ and $h_{\varepsilon} / h_{\mathrm{ex}}$ converges in $W_{\text {loc }}^{1, p}(\Omega)$ to a solution of $(1.2)$ with $c \equiv 1$.

(3) $h_{\mathrm{ex}}=o\left(n_{\varepsilon}\right)$ and then $\mu_{\varepsilon} / n_{\varepsilon}$ converges in $W^{-1, p}(\Omega)$ to a measure $\mu$ and $h_{\varepsilon} / n_{\varepsilon}$ converges in $W_{\text {loc }}^{1, p}(\Omega)$ to the solution of 1.2 with $c \equiv 0$.

In cases (2) and (3), if $\mu \in H^{-1}(\Omega)$ then the solution $h_{\mu} \in H^{1}(\Omega)$ of 1.2 and its associated symmetric stress-energy tensor $T_{\mu} \equiv\left(\left(T_{\mu}\right)_{i j}\right)_{1 \leqslant i, j \leqslant 2}$ defined by

$$
T_{\mu}=\left(\begin{array}{cc}
\frac{1}{2}\left(\left(\partial_{2} h_{\mu}\right)^{2}-\left(\partial_{1} h_{\mu}\right)^{2}+h_{\mu}^{2}\right) & -\partial_{1} h_{\mu} \partial_{2} h_{\mu} \\
-\partial_{1} h_{\mu} \partial_{2} h_{\mu} & \frac{1}{2}\left(\left(\partial_{1} h_{\mu}\right)^{2}-\left(\partial_{2} h_{\mu}\right)^{2}+h_{\mu}^{2}\right)
\end{array}\right)
$$

has the following properties: $T_{\mu}$ is in $L_{\text {loc }}^{1}(\Omega)$,

$$
\operatorname{div} T_{\mu}=0 \text { in the sense of distributions and }\left|\nabla h_{\mu}\right|^{2} \in W_{\text {loc }}^{1, q}(\Omega) \forall q \in[1, \infty) .
$$

The first property of 1.8 means that for $i=1,2$, we have $\partial_{1}\left(T_{\mu}\right)_{i 1}+\partial_{2}\left(T_{\mu}\right)_{i 2}=0$ in the sense of distributions while the latter property implies that $h_{\mu}$ is locally Lipschitz.

Thus, cases (2) and (3) correspond to equation (1.1). These cases are most interesting but not very well understood, especially when $\mu$ is not absolutely continuous with respect to the Lebesgue measure. The main question is to understand the nontriviality and the sign of $\mu$ (recall that $\mu$ is the limiting measure of $\left.\left(2 \pi / n_{\varepsilon}\right) \sum_{i} d_{i}^{\varepsilon} \delta_{a_{i}^{\varepsilon}}\right)$, which in turn gives qualitative information on the behavior of vortices.

In the rest of the section, we will be more specific on this question. In Theorem $1.1, d_{i}^{\varepsilon}$ 's are the degrees of the vortices $a_{i}^{\varepsilon}$ 's; typically $d_{i}^{\varepsilon}= \pm 1$ in stable configurations. On the $\varepsilon$-level, it is expected that $d_{i}^{\varepsilon}$ 's can have different signs and thus the approximating measures $v_{\varepsilon}$ are not necessarily of distinguished sign. When we do the space rescaling as in cases (2) and (3), close vortices of different signs may annihilate each other. This leads us to an open problem (Problem 17 of [11]) about the possibility of having solutions with nonpositive/changing sign limiting measures. Our Corollary 1.1.i) in the next section partially answers this in the negative direction. 
In the above theorem, if all vortices have uniformly bounded degrees (which is physically possible) then the quantity $n_{\varepsilon}$ is basically the total number of vortices of the critical points of the Ginzburg-Landau energy. Case (3) only happens for critical points with a number of vortices $n_{\varepsilon}$ much larger than the applied magnetic field $h_{\mathrm{ex}}$. This leads us to another open problem (Problem 18 in [11]) about the possibility of having critical points with a number of vortices much larger than the applied magnetic field $h_{\mathrm{ex}}$. As we will see in Theorem 1.2 below, for thin domains, the answer to this open problem is negative.

Recall that if $\mu \in L^{p}(\Omega)$ where $p>1$ then the answer to both Problems $17 \& 18$ is negative; this fact was established in Theorem 13.1 of [11].

We note that our assumption on $\mu$ in the context of Ginzburg-Landau vortices corresponds to the case where limiting vortices concentrate on a closed smooth curve in $\Omega$. This type of limiting vorticity can happen. Indeed, recently Aydi [2] showed that when $\Omega$ is the unit disc, a nonzero vorticity $\mu$ which is supported in a finite union of concentric circles can actually arise as limit of the vorticities of some family of solutions.

\subsection{Main results and methods of proof}

Our first result concerns the regularity of $h_{\mu}$ in terms of that of $\mu$.

PROPOSITION 1.1 Suppose that $\mu$ is absolutely continuous with respect to the arclength measure on $\Sigma$ with density $f \in W^{2, p}(\Sigma)$ for some $p>1$, i.e., $\mu=f \mathcal{H}^{1}\left\lfloor\Sigma\right.$. Then the solution $h_{\mu}$ of 1.2 is $C^{1,1-1 / p}$ up to the boundary on each subdomain of $\Omega$ enclosed by $\Sigma$ and/or $\partial \Omega$.

REMARK 1.1 Every $\mu$ as in the above proposition is in $W^{-1, q}(\Omega)$ for all $q>1$.

We note that (1.1)-(1.2) implies (1.8); see, e.g., [11]. Below, we need (1.8) and the following condition on the solution $h_{\mu}$ of $(1.2)$ :

(A) $h_{\mu}$ is $C^{1}$ up to the boundary on each side of $\Sigma$.

Rather than imposing this condition on $h_{\mu}$, we feel it is more natural to impose a condition on the measure $\mu$. This is why we gave a sufficient condition in terms of $\mu$ in Proposition 1.1 By this proposition, $(A)$ is satisfied if $\mu=f \mathcal{H}^{1}\left\lfloor\Sigma\right.$ with $f \in W^{2, p}(\Sigma)$ for some $p>1$.

When $\mu$ is clear from context, the subscript $\mu$ is dropped in $h_{\mu}, T_{\mu}$, etc.

NotAtion We denote by $\lambda_{1}(\Omega)$ the first eigenvalue of the Laplace operator $-\Delta$ on $\Omega$ with zero Dirichlet boundary condition. By $[1.8, T$ is a locally bounded tensor. With the additional assumption $(A)$, its regularity is improved. This is the object of our next result which confirms the continuity of $T$ and the nonsolvability of $(1.1)-1.2$ for nonzero measures $\mu$ on a thin domain.

THEOREM 1.2 Assume that $(A)$ is satisfied. Then

(i) $T$ is continuous in $\Omega$ and $h$ is equal to a constant $c_{*}$ on $\Sigma$.

(ii) If either $\lambda_{1}(\Omega)>1$ or $\operatorname{diam}(\Omega)|\Omega|^{1 / 2} \leqslant 2 \pi^{1 / 2}$ then $[1.1-1.2$ with $c \equiv 0$ has no solution with a nonzero measure $\mu \in H^{-1}(\Omega)$.

(iii) If $\Omega=B_{R}$ then 1.1 - 1.2 with $c \equiv 0$ has no solution with a nonzero measure $\mu \in H^{-1}(\Omega)$ supported on a circle.

For the case $\mu \in L^{p}(\Omega)$ for some $p>1$ in case (2) of Theorem 1.1 it can be shown that the measure $\mu$ is in fact given by a nonnegative $L^{\infty}$ function. In contrast, in our case with a more 
singular vorticity measure $\mu$, there is no gain in the regularity of $\mu$, except its density with respect to the arclength measure on $\Sigma$. However, there is a gain in regularity for the stress-energy tensor $T$ and on $h$. As a simple consequence of the constancy of $h$ on $\Sigma$, there is also an improvement in the regularity of $h$ on each side of $\Sigma: h$ is $C^{\infty}$ up to the boundary on each side of $\Sigma$. Furthermore, $\mu$ is absolutely continuous with respect to the arclength measure on $\Sigma$ with smooth density $f \in C^{\infty}(\Sigma)$ (see (3.6)). In addition, we obtain further information on the sign of $\mu$ in the following

COROLlary 1.1 Suppose that $(1.1)-(1.2)$ and $(A)$ are satisfied. Then

(i) $\mu$ is a Radon measure with a fixed sign.

(ii) If $\mu$ is a measure with constant density on $\Sigma$, then $\Sigma$ is a circle.

(iii) If, in addition, $h=1$ on $\partial \Omega$ and $\Omega$ is thin enough, i.e., $\lambda_{1}(\Omega)>1$ or $\operatorname{diam}(\Omega)|\Omega|^{1 / 2} \leqslant 2 \pi^{1 / 2}$, then $\mu$ is a positive Radon measure.

Let us say a few words about the methods of proof. The proof of Proposition 1.1 is straightforward from potential-theoretic arguments. For the proof of part (i) of Theorem 1.2 the key idea is to express the stress-energy tensor $T$ in the normal-tangential frame $(\nu, \tau)$ associated with $\Sigma$. In this frame, due to the smoothness of $\Sigma$ and assumption $(A)$, we have the regularity of $T$ in the tangential direction. What prevents $T$ from being continuous is the jump in the normal derivative of $h$. At this point we use the divergence-free property of $T$ to show that its normal components are preserved and thus continuous through $\Sigma$. The constancy of $h$ on $\Sigma$ is crucial in the proof of parts (ii) and (iii) of Theorem 1.2 . It allows us to reflect part of the graph of the function $h$ in $\Omega^{-}$, the region enclosed by $\Sigma$, over the horizontal plane $z=c_{*}$ in $\mathbb{R}^{3}$. By this reflection, we obtain equation (3.10) which is less singular than (1.2). Then we can use some type of Aleksandrov weak maximum principle and the modified Bessel functions to conclude.

The paper is organized as follows. In Section 2 we prove Proposition 1.1 The proof of Theorem 1.2 and Corollary 1.1 is presented in Section 3 . Our study leaves as many problems open as it solves. We present a list of open questions and conjectures in Section 4.

\section{Proof of Proposition 1.1}

Before proving this proposition, we make a few simplifications. First, we can assume that $c=0$. Indeed, if $c=1$ then we can split $h=h_{0}+h_{1}$ where $h_{0}$ and $h_{1}$ solve the following equations respectively:

$$
-\Delta h_{0}+h_{0}=\mu \quad \text { in } \Omega, \quad h_{0}=0 \quad \text { on } \partial \Omega
$$

and

$$
-\Delta h_{1}+h_{1}=0 \quad \text { in } \Omega, \quad h_{1}=1 \quad \text { on } \partial \Omega .
$$

Because $h_{1} \in C^{\infty}(\bar{\Omega})$ by standard regularity theory, the regularity of $h$ is that of $h_{0}$.

Second, we only need to establish the regularity results for the simpler equation

$$
\begin{cases}-\Delta u=\mu & \text { in } \Omega \\ u=0 & \text { on } \partial \Omega\end{cases}
$$

To see this, recall that by Remark 1.1 we have $\mu \in W^{-1,2 p}(\Omega)$ and therefore, by elliptic regularity, $h \in W^{1,2 p}(\Omega)$. Let $v$ solve $-\Delta v=-h$ in $\Omega$ with $v=0$ on $\partial \Omega$. Then $v \in W^{3,2 p}(\Omega)$ and thus 
$v \in C^{2,1-1 / p}(\Omega)$. Let $H=h-v$. Then $H$ is the solution to 2.1 ). Therefore, the regularity (in $C^{1}$ ) of $h$ is the same as that of $H$. From now on, by $h$ we mean the solution to 2.1).

Third, we can assume that the smooth curve $\Sigma$, the support of $\mu$, is a circle. This follows from the fact that the regularity of an elliptic equation is unaltered through a conformal change of variables. Let $\Omega^{-}$be the subdomain of $\Omega$ enclosed by $\Sigma$. We can find a smooth map $(x, y) \in$ $B_{r} \mapsto(f(x, y), g(x, y)) \equiv(X, Y) \in \Omega^{-}$with $\partial f / \partial x=\partial g / \partial y, \partial f / \partial y=-\partial g / \partial x$. In $B_{r}$, let $\tilde{h}(x, y)=h(X, Y)=h(f(x, y), g(x, y))$. Then a simple calculation gives

$$
-\Delta \tilde{h}(x, y)=\Delta h(X, Y)|\nabla f|^{2}=\mu(X, Y)|\nabla f|^{2}=\tilde{\mu}(x, y) .
$$

Note that $\mathrm{d} \mu /\left(\mathrm{d} \mathcal{H}^{1}\lfloor\Sigma)\right.$ and $\mathrm{d} \tilde{\mu} /\left(\mathrm{d} \mathcal{H}^{1}\left\lfloor\partial B_{r}\right)\right.$ have the same smoothness.

Finally, we can assume that $\Omega$ and $B_{r}$ are concentric discs. Indeed, let $B_{R} \ni \Omega$ and let $u$ solve $-\Delta u=\mu$ in $B_{R}, u=0$ on $\partial B_{R}$. Because supp $\mu \Subset \Omega \Subset B_{R}$, we find that $u$ is $C^{\infty}$ in $B_{R} \backslash \bar{\Omega}$ up to its boundary. Thus $u=g \in C^{\infty}(\partial \Omega)$ on $\partial \Omega$. We have

$$
\begin{cases}-\Delta(u-h)=0 & \text { in } \Omega, \\ u-h=g & \text { on } \partial \Omega .\end{cases}
$$

By elliptic regularity, $u-h \in C^{\infty}(\bar{\Omega})$. Thus, the regularity of $h$ is the same as that of $u$. Consequently, by scaling, we assume that $\Omega$ is the unit disc, i.e., $\Omega=B_{1}$.

In conclusion, we only need to establish the regularity results for the simplest equation

$$
\begin{cases}-\Delta u=\mu & \text { in } B_{1}, \\ u=0 & \text { on } \partial B_{1},\end{cases}
$$

with $\mu$ supported on a circle, say, $\partial B_{r}(0<r<1)$.

As a preparation for the proof of Proposition 1.1, we prove the following

LEMMA 2.1 If $\mathrm{d} \mu(y)=f(y) \mathrm{d} \mathcal{H}^{1}\left\lfloor\partial B_{r}(0<r<1)\right.$ where $f \in W^{2, p}\left(\partial B_{r}\right)(p>1)$ then the function $k$ defined by $k(x)=\int \log |x-y| \mathrm{d} \mu(y)$ belongs to $W^{2, p}\left(\partial B_{r}\right)$ when restricted to $\partial B_{r}$.

Proof. For $x \in \partial B_{r}$, we write $x=r e^{i t}(0 \leqslant t<2 \pi)$ and define

$$
k(t)=k(x)=\int \log \left|r e^{i t}-y\right| \mathrm{d} \mu(y) .
$$

Then, writing $y=r e^{i \varphi}(0 \leqslant \varphi<2 \pi)$ for $y \in \partial B_{r}$, we have

$$
\left|r e^{i t}-y\right|^{2}=\left|r e^{i t}-r e^{i \varphi}\right|^{2}=r^{2}\left|1-e^{i(t-\varphi)}\right|^{2}=2 r^{2}(1-\cos (t-\varphi))
$$

and therefore

$$
\begin{aligned}
k(t) & =\frac{1}{2} \int \log |x-y|^{2} \mathrm{~d} \mu(y)=\frac{1}{2} \int_{\partial B_{r}} \log |x-y|^{2} \mathrm{~d} \mu(y) \\
& =\frac{1}{2} \int \log \left(2 r^{2}(1-\cos (t-\varphi))\right) f(\varphi) r \mathrm{~d} \varphi \\
& =\frac{1}{2} \int \log (1-\cos (t-\varphi)) f(\varphi) r \mathrm{~d} \varphi+\frac{1}{2} \int \log \left(2 r^{2}\right) f(\varphi) r \mathrm{~d} \varphi
\end{aligned}
$$


It follows that the regularity of $k$ is that of $g(t)=\int \log (1-\cos (t-\varphi)) f(\varphi) \mathrm{d} \varphi$. We can rewrite $g$ as $g(t)=w * f(t)$ where $w(s)=\log (1-\cos (s)) \in L^{1}(0,2 \pi)$. Now, if $f \in W^{2, p}\left(\partial B_{r}\right)$ then $g \in W^{2, p}(0,2 \pi)$ and thus, when restricted to $\partial B_{r}, k \in W^{2, p}\left(\partial B_{r}\right)$ as claimed.

Now, after simplifications, the proof of Proposition 1.1 is reduced to that of the following

PROPOSITION 2.1 Let $\mathrm{d} \mu(y)=f(y) \mathrm{d} \mathcal{H}^{1}\left\lfloor\partial B_{r}(0<r<1)\right.$ where $f \in W^{2, p}\left(\partial B_{r}\right)(p>1)$. Then the solution to the equation 2.2 is $C^{1,1-1 / p}$ up to the boundary on each subdomain of $\Omega$ enclosed by $\partial B_{r}$ and/or $\partial B_{1}$.

Proof. We have $u(x)=\int G(x, y) \mathrm{d} \mu(y)$ where $G$ is the Green function associated with $B_{1}$ :

$$
-\Delta_{y} G=\delta_{x} \quad \text { in } B_{1}, \quad G=0 \quad \text { on } \partial B_{1} .
$$

An explicit formula for $G(x, y)$ (see, e.g., [7]) is given by

$$
\begin{aligned}
G(x, y) & =-\frac{1}{2 \pi} \log |x-y|+\frac{1}{2 \pi} \log || x\left|\left(y-\frac{x}{|x|^{2}}\right)\right| \\
& =-\frac{1}{2 \pi} \log |x-y|+\frac{1}{2 \pi} \log \sqrt{|x|^{2}|y|^{2}-2 x \cdot y+1} .
\end{aligned}
$$

Thus

$$
\begin{aligned}
u(x) & =-\frac{1}{2 \pi} \int \log |x-y| \mathrm{d} \mu(y)+\frac{1}{4 \pi} \int \log \left(|x|^{2}|y|^{2}-2 x \cdot y+1\right) \mathrm{d} \mu(y) \\
& =-\frac{1}{2 \pi} k(x)+\text { smooth function in } x .
\end{aligned}
$$

By Lemma 2.1, when restricted to $\partial B_{r}, k$ is in $W^{2, p}$ on $\partial B_{r}$ and so is $u$. Therefore $u \in C^{1,1-1 / p}$ on $\partial B_{r}$. By a standard regularity result (see, e.g., [6]), the proposition follows.

\section{Proofs of Theorem 1.2 and Corollary 1.1}

Let $\Omega^{-}$(resp. $\Omega^{+}$) be the region in $\Omega$ enclosed by $\Sigma$ (resp. by $\Sigma$ and $\partial \Omega$ ). On $\Sigma$, we choose the unit normal vector $v$ pointing into $\Omega^{+}$. In the normal-tangential frame $(\nu, \tau)$, we have

$$
T=\left(\begin{array}{ll}
T_{\nu \nu} & T_{\nu \tau} \\
T_{\tau \nu} & T_{\tau \tau}
\end{array}\right)=\left(\begin{array}{cc}
\frac{1}{2}\left(\left(\partial_{\tau} h\right)^{2}-\left(\partial_{\nu} h\right)^{2}+h^{2}\right) & -\partial_{\nu} h \partial_{\tau} h \\
-\partial_{\nu} h \partial_{\tau} h & \frac{1}{2}\left(\left(\partial_{\nu} h\right)^{2}-\left(\partial_{\tau} h\right)^{2}+h^{2}\right)
\end{array}\right) .
$$

Proof of Theorem 1.2(i). By assumption $(A)$, the tangential derivative $\partial_{\tau} h$ exists and is continuous on $\Sigma$, and in $\Omega^{-}$with the frame $(\nu, \tau)$, the normal derivative $\partial_{\nu} h$ exists and is continuous up to its boundary $\Sigma$. Therefore, $T$ is continuous on each side of $\Sigma$ up to the curve $\Sigma$.

First, we prove the continuity of $T$ through $\Sigma$. Indeed, since $h$ is smooth off $\Sigma, T$ has $\Sigma$ as its only curve of discontinuity. Denote by $T^{+}(\Sigma)$ (resp. $T^{-}(\Sigma)$ ) the trace of $\left.T\right|_{\Omega^{+}}$(resp. $\left.T\right|_{\Omega^{-}}$) on $\Sigma$. By the divergence-free property of $T$, we have

$$
\int_{\Omega} T \cdot \nabla \varphi=0
$$


for all test functions $\varphi \in C_{c}^{1}(\Omega)$. We now consider test functions $\varphi$ whose supports cross $\Sigma$. Denote by $[T]=T^{+}(\Sigma)-T^{-}(\Sigma)$ the jump of $T$ across $\Sigma$. Then, integrating by parts the equation 3.1 , we see that

$$
0=\int_{\Omega} T \cdot \nabla \varphi=-\int_{\Omega^{+}}(\operatorname{div} T) \varphi-\int_{\Omega^{-}}(\operatorname{div} T) \varphi-\int_{\Sigma} \varphi[T] \cdot v \mathrm{~d} \mathcal{H}^{1}
$$

where $\mathcal{H}^{1}$ denotes the 1-dimensional Hausdorff measure. On the right hand side, the first two terms are zero by the divergence-free property of $T$ in the strong sense on each side of the curve $\Sigma$; hence the last term must vanish. Because $\varphi$ is arbitrary, this implies that $[T] \cdot v=0$, meaning that there is no jump in the normal components of $T$. Therefore, the normal components of $T$, $T_{\nu \nu}=\left(\partial_{\tau} h\right)^{2}-\left(\partial_{\nu} h\right)^{2}+h^{2}$ and $T_{\tau \nu}=\partial_{\nu} h \partial_{\tau} h$, are continuous through $\Sigma$. Recall that $(1.1)-$ 1.2 implies (1.8) and thus $h$ is continuous through $\Sigma$. Hence, it follows that $\left(\partial_{\tau} h\right)^{2}-\left(\partial_{\nu} h\right)^{2}$ is continuous through $\Sigma$, showing that $T$ is continuous through $\Sigma$.

Next, we prove the constancy of $h$ on $\Sigma$. Let $h^{ \pm}$be the restrictions of $h$ on $\Omega^{ \pm}$. Then we can rewrite the equation $\mu=-\Delta h+h$ in terms of $h^{ \pm}$as follows:

$$
\mu=\left(\frac{\partial h^{-}}{\partial v}-\frac{\partial h^{+}}{\partial v}\right) \mathrm{d} \mathcal{H}^{1}\lfloor\Sigma .
$$

By $(1.8),|\nabla h|^{2}$ is continuous and as proved above, $\left(\partial_{\tau} h\right)^{2}-\left(\partial_{\nu} h\right)^{2}$ is continuous through $\Sigma$. Thus, $\left(\partial_{\tau} h\right)^{2}$ and $\left(\partial_{\nu} h\right)^{2}$ are continuous through $\Sigma$. Consequently, on $\Sigma$, we have

$$
\frac{\partial h^{-}}{\partial v}= \pm \frac{\partial h^{+}}{\partial v}
$$

Because $\mu$ has a nowhere-zero density on $\Sigma$, we deduce from 3.3 and 3.4 that

$$
\frac{\partial h^{-}}{\partial v}=-\frac{\partial h^{+}}{\partial v} \neq 0
$$

and that

$$
\mu=2 \frac{\partial h^{-}}{\partial v} \mathrm{~d} \mathcal{H}^{n-1}\lfloor\Sigma .
$$

Since $\partial_{\nu} h \partial_{\tau} h$ is continuous through $\Sigma$ and $\partial_{\nu} h$ changes its sign through $\Sigma$, we must have $\partial_{\tau} h=0$ on $\Sigma$. This allows us to conclude that $h$ is equal to a constant $c_{*}$ on $\Sigma$.

Proofs of parts (i) and (ii) of Corollary 1.1. (i) We first prove the nonchanging sign character of $\mu$. By 3.6, it suffices to prove that $\partial h^{-} / \partial v$ does not change sign, but this is a consequence of the constancy of $h^{-}$on $\Sigma$ and Hopf's Lemma.

(ii) Suppose that $\mu$ is a measure with constant density on $\Sigma$. Then, from 3.6 , it follows that $\partial h^{-} / \partial v$ is a nonzero constant on $\Sigma$. Therefore, $h^{-}$solves the following overdetermined system:

$$
-\Delta h^{-}+h^{-}=0 \quad \text { in } \Omega^{-}, \quad h^{-}=\text {constant on } \partial \Omega^{-}, \quad \frac{\partial h^{-}}{\partial v}=\text { constant } \neq 0 \quad \text { on } \partial \Omega^{-} .
$$

By a celebrated theorem of Serrin [12], we conclude that $\Omega^{-}$is a disc and thus $\Sigma$ is a circle. 
Proof of parts (ii) and (iii) of Theorem 1.2 We argue by contradiction. Suppose that 1.1 - 1.2 with $c \equiv 0$ has a solution $h_{\mu}$ for a nonzero measure $\mu \in H^{-1}(\Omega)$. Recall by Corollary 1.1(i) that $\mu$ is a Radon measure with a fixed sign. Because the boundary condition of $h$ in $(1.2)$ is 0 , we can assume without loss of generality that $\mu$ is a positive Radon measure. Thus, by the maximum principle,

$$
0<h^{+}<c_{*} \quad \text { in } \Omega^{+}, \quad 0<h^{-}<c_{*} \quad \text { in } \Omega^{-} .
$$

The proof will be easier once we reflect part of the graph of the function $h$ in $\Omega^{-}$over the horizontal plane $z=c_{*}$ in $\mathbb{R}^{3}$. More precisely, we consider the function $v$ defined as follows:

$$
v= \begin{cases}2 c_{*}-h^{-} & \text {in } \Omega^{-}, \\ h^{+} & \text {in } \Omega^{+} .\end{cases}
$$

Then, as in the proof of Theorem $1.2(\mathrm{i})$, we have the following identity on $\Sigma$ :

$$
-\Delta v+v=\left(\frac{\partial v^{-}}{\partial v}-\frac{\partial v^{+}}{\partial v}\right) \mathrm{d} \mathcal{H}^{n-1}\left\lfloor\Sigma=\left(\frac{-\partial h^{-}}{\partial v}-\frac{\partial h^{+}}{\partial v}\right) \mathrm{d} \mathcal{H}^{n-1}\lfloor\Sigma=0 .\right.
$$

The last equality follows from 3.5. Thus, by a simple calculation, we get

$$
-\Delta v+v=2 c_{*} \chi_{\Omega^{-}} .
$$

By (3.7), we have $0<v<c_{*}$ in $\Omega^{+}, v=c_{*}$ on $\Sigma$, and $c_{*}<v<2 c_{*}$ in $\Omega^{-}$. Therefore, $\Omega^{-}=\left\{v>c_{*}\right\}$. Dividing both sides of 3.10$\}$ by $c_{*}$, we can assume that $c_{*}=1$ and the equation 3.10) can be rewitten as

$$
-\Delta v+v=2 \chi\{v>1\} .
$$

The assertion of Theorem 1.2 (ii) with the thinness assumption is now a consequence of the following PRoposition 3.1 If either $\lambda_{1}(\Omega)>1$ or $\operatorname{diam}(\Omega)|\Omega|^{1 / 2} \leqslant 2 \pi^{1 / 2}$ then the equation

$$
\begin{cases}-\Delta v+v=2 \chi_{\{v>1\}} & \text { in } \Omega, \\ v=0 & \text { on } \partial \Omega,\end{cases}
$$

has no nontrivial solution.

Proof. Suppose by contradiction that there exists such a nontrivial solution. Then $w=\{v>1\}$ has positive Lebesgue measure. By the maximum principle, $v>0$ in $\Omega$.

CASE $1: \lambda_{1}(\Omega)>1$. Let $\varphi>0$ be an eigenvector associated with $\lambda:=\lambda_{1}(\Omega)$. Then

$$
\begin{aligned}
\int_{w} 2 \varphi \mathrm{d} x=\int_{\Omega} 2 \chi_{\{v>1\}} \varphi \mathrm{d} x & =\int_{\Omega}(-\Delta v+v) \varphi \mathrm{d} x=\int_{\Omega}(-\Delta \varphi+\varphi) v \mathrm{~d} x=\int_{\Omega}(\lambda+1) \varphi v \mathrm{~d} x \\
& \geqslant \int_{w}(\lambda+1) \varphi v \mathrm{~d} x \geqslant(\lambda+1) \int_{w} \varphi \mathrm{d} x>2 \int_{w} \varphi \mathrm{d} x .
\end{aligned}
$$

This is impossible because $\varphi>0$ and $|w|>0$.

CASE 2: $\operatorname{diam}(\Omega)|\Omega|^{1 / 2} \leqslant 2 \pi^{1 / 2}$. In this case $v$ solves the equation $-\Delta v=f$ where $f=$ $2 \chi_{\{v>1\}}-v$. Observe that $\|f\|_{L^{\infty}(\Omega)} \leqslant 1$. Therefore, $v \in C^{0}(\bar{\Omega}) \cap W_{\text {loc }}^{2,2}(\Omega)$ with $\sup _{\Omega} v>1$ by the nontriviality of $v$. We are going to use the following weak maximum principle of A. D. Aleksandrov to find a contradiction: 
LEMma 3.1 ([7], Lemma 9.3]) For $u \in C^{2}(\Omega) \cap C^{0}(\bar{\Omega})$, we have

$$
\sup _{\Omega} u \leqslant \sup _{\partial \Omega} u+\frac{\operatorname{diam}(\Omega)}{2 \pi^{1 / 2}}\|\Delta u\|_{L^{2}\left(\Gamma_{u}^{+}\right)} .
$$

In the above lemma, $\Gamma_{u}^{+}$is the upper contact set of the continuous function $u$. It is defined as the subset of $\Omega$ where the graph of $u$ lies below a support hyperplane in $\mathbb{R}^{3}$, i.e.

$$
\Gamma_{u}^{+}=\left\{y \in \Omega \mid u(x) \leqslant u(y)+p .(x-y) \text { for all } x \in \Omega \text { for some } p=p(y) \in \mathbb{R}^{2}\right\} .
$$

In view of 3.13 and the fact that $\Gamma_{u}^{+} \subset \Omega$, one finds that for $u \in C^{2}(\Omega) \cap C^{0}(\bar{\Omega})$,

$$
\sup _{\Omega} u \leqslant \sup _{\partial \Omega} u+\frac{\operatorname{diam}(\Omega)}{2 \pi^{1 / 2}}\|\Delta u\|_{L^{2}(\Omega)} .
$$

By an approximation argument, we can extend the above inequality to functions $u \in C^{0}(\bar{\Omega}) \cap$ $W_{\text {loc }}^{2,2}(\Omega)$. Now applying 3.14 to our function $v$, we obtain

$$
1<\sup _{\Omega} v \leqslant \sup _{\partial \Omega} v+\frac{\operatorname{diam}(\Omega)}{2 \pi^{1 / 2}}\|\Delta v\|_{L^{2}(\Omega)}=\frac{\operatorname{diam}(\Omega)}{2 \pi^{1 / 2}}\|f\|_{L^{2}(\Omega)} \leqslant \frac{\operatorname{diam}(\Omega)}{2 \pi^{1 / 2}}|\Omega|^{1 / 2} \leqslant 1,
$$

which is clearly a contradiction completing the proof of Proposition 3.1

REMARK 3.1 For the unit disc $B_{1}$, we have $\lambda_{1}\left(B_{1}\right)=\left(J_{0}(1)\right)^{2}>5$ where $J_{0}(x)$ is the Bessel function of the first kind.

The assertion of Theorem 1.2 (iii) is confirmed by the following

Proposition 3.2 For any disc domain $\Omega=B_{R}$, the equation 3.12 has no nontrivial solution such that $w=\{v>1\}$ is a disc $B_{r}\left(x_{0}\right)$.

Proof. If $w$ is a disc, then from Theorem 5 in Sirakov [13], it follows that $\Omega$ and $w$ are concentric. Therefore, $x_{0}=0$ and $w=B_{r}$. It is now convenient to switch back to the potential function $h$. Because $h^{-}$and $h^{+}$are respectively the solutions to the equation $-\Delta h+h=0$ in $B_{r}$ and $B_{R} \backslash B_{r}$, they must be radial: $h^{-}(x)=g^{-}(|x|)$ and $h^{+}(x)=g^{+}(|x|)$. For radial functions $h(x)=g(|x|)$, the equation $-\Delta h+h=0$ reads

$$
-g^{\prime \prime}(|x|)-\frac{g^{\prime}(|x|)}{|x|}+g(|x|)=0 .
$$

This equation can be solved with the help of the modified Bessel functions as in Aydi [2]. We define $I_{0}$ and $K_{0}$ to be respectively the modified Bessel function of the first kind and of the second kind:

$$
I_{0}(x)=\sum_{n=0}^{\infty} \frac{x^{2 n}}{(n !)^{2} 2^{2 n}}, \quad K_{0}(x)=-(\log (x / 2)+\gamma) I_{0}(x)+\sum_{n=0}^{\infty} \frac{x^{2 n}}{(n !)^{2} 2^{2 n}} \phi(n),
$$

where $\phi(n)=\sum_{k=1}^{n} 1 / k$ for $n \neq 0, \phi(0)=0$, and $\gamma=\lim _{n \rightarrow \infty}(\phi(n)-\log n)$. These functions are continuous solutions to the ODE

$$
-y^{\prime \prime}-\frac{y^{\prime}}{x}+y=0 \quad \text { for } 0 \leqslant x<\infty
$$

with a singularity at 0 for $K_{0}$. See Watson [14] for the alternative definitions and properties. Define $I_{1}$ and $K_{1}$ to be respectively the derivative of $I_{0}$ and $-K_{0}$. 
Now, we can use the modified Bessel functions to find $h^{-}, g^{-}, h^{+}$and $g^{+}$. Clearly, they have the forms

$$
h^{-}(x)=g^{-}(|x|)=a I_{0}(|x|) \quad \text { in } B_{r}, \quad h^{+}(x)=g^{+}(|x|)=b I_{0}(|x|)+c K_{0}(|x|) \quad \text { in } B_{R} \backslash B_{r}
$$

where $a, b, c$ are constants to be determined. Let us use the compatibility conditions on $\Sigma=\partial B_{r}$ to find the relations between $a, b, c$. First, by the continuity of the function $h$ on $\Sigma$, we require that $g^{-}(r)=g^{+}(r)$, which is equivalent to

$$
a I_{0}(r)=b I_{0}(r)+c K_{0}(r) .
$$

Second, by the jump condition (3.5) of the normal derivative of the function $h$ on $\Sigma$, we must have $\frac{\partial g^{-}}{\partial v}(r)=-\frac{\partial g^{+}}{\partial v}(r)$, which is equivalent to

$$
a I_{1}(r)=-b I_{1}(r)+c K_{1}(r) .
$$

Finding $a, b$ in terms of $c$ from these equations, we obtain

$$
a=\frac{c}{2}\left(\frac{K_{1}(r)}{I_{1}(r)}+\frac{K_{0}(r)}{I_{0}(r)}\right), \quad b=\frac{c}{2}\left(\frac{K_{1}(r)}{I_{1}(r)}-\frac{K_{0}(r)}{I_{0}(r)}\right) .
$$

Observe the following property of Bessel functions:

$$
\frac{\mathrm{d}}{\mathrm{d} x}\left(-K_{0}(x) I_{0}(x)\right)=K_{1}(x) I_{0}(x)-I_{1}(x) K_{0}(x)>0 \quad \text { for all } x>0 .
$$

Thus, $a, b, c$ have the same sign. Because $h^{-}>0$, it follows that $a, b, c>0$. Thus $h^{+}(R)>0$. This is a contradiction with the zero Dirichlet boundary condition of $h$.

Proof of Corollary 1.1 (iii). Suppose otherwise that $\mu$ is a negative Radon measure. Then we must have $c_{*}<0$. Indeed, by (3.6) and the negativity of $\mu$, we find that $\partial h^{-} / \partial v<0$. Therefore, $h^{-}$ achieves its maximum at a point $x_{0}$ inside $\Omega^{-}$. Note also that $h^{-}$satisfies $-\Delta h^{-}+h^{-}=0$ in $\Omega^{-}$. Consequently, $c_{*}<h^{-}\left(x_{0}\right)=\Delta h^{-}\left(x_{0}\right) \leqslant 0$. Consider the function $h^{+}$. It satisfies $-\Delta h^{+}+h^{+}=0$ in the domain $\Omega^{+}$and has boundary values $h^{+}=c_{*}<0$ on $\Sigma$ and $h^{+}=1$ on $\partial \Omega$. Therefore, $h^{+}$ is smooth in $\overline{\Omega^{+}}$and we can find a smooth closed curve $\Sigma^{\prime} \subset\left\{x \in \Omega^{+} \mid h^{+}(x)=0\right\}$ in $\Omega^{+}$. Let $\Omega^{\prime}$ be the subdomain of $\Omega$ with boundary $\partial \Omega^{\prime}=\Sigma^{\prime}$. Then, by the thinness assumption on $\Omega$, we also have the thinness property of $\Omega^{\prime}$, i.e., $\lambda_{1}\left(\Omega^{\prime}\right)>1$ or $\operatorname{diam}\left(\Omega^{\prime}\right)\left|\Omega^{\prime}\right|^{1 / 2} \leqslant 2 \pi^{1 / 2}$. Now, consider the function $h^{\prime}=\left.h\right|_{\Omega^{\prime}}$. It solves

$$
\begin{cases}-\Delta h^{\prime}+h^{\prime}=\mu \neq 0 & \text { in } \Omega^{\prime}, \\ h^{\prime}=0 & \text { on } \partial \Omega,\end{cases}
$$

with $\operatorname{div} T^{\prime}=0$ where $T^{\prime}$ is the stress-energy tensor associated with $h^{\prime}$ defined similarly to 1.7 . This is impossible by Theorem 1.2 ii) proved above. Thus $\mu$ must be a positive Radon measure.

\section{Perspectives and open problems}

\subsection{Optimal regularity and maximum principle}

It was proved in [10] that if the limiting vorticity $\mu$ is in $L^{p}(\Omega)$ for some $p>1$ then the function $h$ solving $1.1-1.2$ is in $C^{1, \alpha}(\Omega)$ for all $\alpha<1$, and furthermore $0 \leqslant h \leqslant 1$ if its boundary value 
is 1 . These conclusions are not true anymore if $\mu$ is only in $H^{-1}(\Omega)$. The Lipschitz continuity of $h$ in this case is optimal as can be seen from the jump relation $(3.5)$ for the normal derivatives of $h$ on each side of $\Sigma$. However, the reflected graph of $h$ is $C^{1, \alpha}(\Omega)$ for all $\alpha<1$. Indeed, this reflected graph is just the graph of the function $v$ defined in (3.8). Because $v$ solves $-\Delta v+v=2 c_{*} \chi_{\Omega^{-}}$in $\Omega$ with constant boundary value $v=c$ on $\partial \Omega$, by standard $L^{p}$-estimates, we have $v \in W^{2, p}(\Omega)$ for all $p>1$. By Sobolev embedding, we conclude that $v \in C^{1, \alpha}(\Omega)$ for all $\alpha<1$.

Now, we show by an example that the bounds $0 \leqslant h \leqslant 1$ fail for $\mu \in H^{-1}(\Omega)$. We prove the following

PROPOSITION 4.1 Let $\Omega=B_{1}$. Then there exists a largest number $M>1$ such that for all $c_{*} \in\left(1 / I_{0}(1), M\right]$, we can find $r \in(0,1)$ and a vorticity measure $\mu$ concentrated on $\partial B_{r}$ with the following property: the function $h_{\mu}$ solving (1.1)-1.2) with $c \equiv 1$ satisfies $h_{\mu}=c_{*}$ on $\partial B_{r}$.

Proof. We use the same notations as in the proof of Proposition 3.2 We are looking for a function $h$ of the form

$$
h^{-}(x):=h(x)=a I_{0}(|x|) \text { in } B_{r}, \quad h^{+}(x):=h(x)=b I_{0}(|x|)+c K_{0}(|x|) \text { in } B_{1} \backslash B_{r},
$$

where $a, b, c, r$ are to be determined such that

$$
a I_{0}(r)=b I_{0}(r)+c K_{0}(r)=c^{*}, \quad \frac{\partial h^{-}}{\partial v}(r)=-\frac{\partial h^{+}}{\partial v}(r), b I_{0}(1)+c K_{0}(1)=1 .
$$

As in 3.15, we now find $b, c$ in terms of $a$ from the first two equations in (4.1) to obtain

$$
\left\{\begin{array}{l}
b=a \frac{K_{1}(r) I_{0}(r)-I_{1}(r) K_{0}(r)}{I_{0}(r) K_{1}(r)+K_{0}(r) I_{1}(r)}=\frac{c^{*}}{I_{0}(r)} \frac{K_{1}(r) I_{0}(r)-I_{1}(r) K_{0}(r)}{I_{0}(r) K_{1}(r)+K_{0}(r) I_{1}(r)}, \\
c=2 a \frac{I_{0}(r) I_{1}(r)}{I_{0}(r) K_{1}(r)+K_{0}(r) I_{1}(r)}=\frac{2 c^{*}}{I_{0}(r)} \frac{I_{0}(r) I_{1}(r)}{I_{0}(r) K_{1}(r)+K_{0}(r) I_{1}(r)} .
\end{array}\right.
$$

Plugging these values of $b$ and $c$ into the last equation in (4.1), we get

$$
q(r):=\frac{1}{I_{0}(r)} \frac{\left\{K_{1}(r) I_{0}(r)-I_{1}(r) K_{0}(r)\right\} I_{0}(1)+2 I_{0}(r) I_{1}(r) K_{0}(1)}{I_{0}(r) K_{1}(r)+K_{0}(r) I_{1}(r)}=\frac{1}{c^{*}} .
$$

Note that for modified Bessel functions (see [14, p. 80]), $I_{0}(r) K_{1}(r)+K_{0}(r) I_{1}(r)=1 / r$. Thus

$$
q(r)=r \frac{\left\{K_{1}(r) I_{0}(r)-I_{1}(r) K_{0}(r)\right\} I_{0}(1)+2 I_{0}(r) I_{1}(r) K_{0}(1)}{I_{0}(r)} .
$$

From the graph of $q$ (see Figure 1 , the proposition easily follows; observe that $\min _{0 \leqslant r \leqslant 1} q(r) \leqslant$ $q(0.8)<1$ and thus we can choose $M=1 / \min _{0 \leqslant r \leqslant 1} q(r)$.

REMARK 4.1 For $c_{*} \in(1, M)$, there are exactly two pairs $(r, \mu)$ where $r \in(0,1)$ and the vorticity measure $\mu$ concentrated on $\partial B_{r}$ with the property: the function $h$ solving (1.1)-(1.2) with $c \equiv 1$ satisfies $h=c_{*}$ on $\partial B_{r}$. This is a rather unexpected fact.

\subsection{Links to the Euler equation and free boundary problems}

Equation (1.1)-1.2 serves as a stationary mean-field model (also called hydrodynamic limit) for superconducting materials and it has many common features with the Euler equations for incompressible flow in fluid mechanics. Consider the 2D Euler equation:

$$
\partial_{t} v+\operatorname{div}(v \otimes v)+\nabla p=0, \quad \operatorname{div} v=0 .
$$




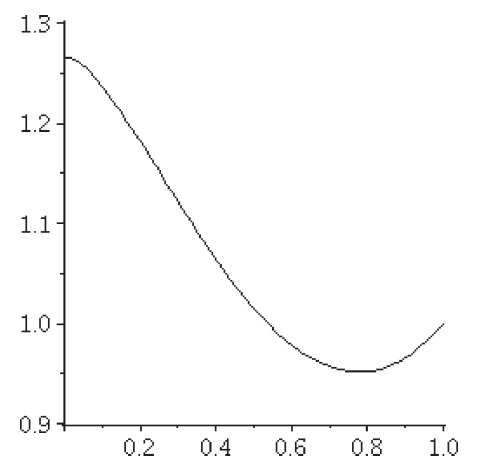

FIG. 1. The graph of $q$

Introduce the stream function $\Psi$ such that $v=\nabla^{\perp} \Psi$. Then the vorticity becomes

$$
w=\operatorname{curl} v=\operatorname{curl}\left(\nabla^{\perp} \Psi\right)=\Delta \Psi .
$$

In our equation, $\mu$ plays the role of $w$ and $h$ plays the role of $\Psi$. If $\mu \in L^{p}(\Omega)$ for some $p>1$ then it was proved in [11, Theorem 13.1] that for $c \equiv 1$ we have $0 \leqslant h_{\mu} \leqslant 1$ and $\mu$ is a nonnegative $L^{\infty}$ function: $\mu=h_{\mu} \chi_{\left\{\left|\nabla h_{\mu}\right|=0\right\}}$. Thus $\mu$ is constant on each "patch" in $\Omega$. This is reminiscent of the vortex patch problem. In this case, equation 1.2 becomes

$$
-\Delta h+h=h \chi\{|\nabla h|=0\}
$$

and its free-boundary regularity was investigated by Caffarelli-Salazar-Shahgholian [4].

When the limiting vorticity $\mu$ is supported on a curve, it has many common features with its counterpart in the Euler equations: the vortex sheet. For more information on this, we refer to Chapters 9-11 of Majda and Bertozzi [9].

\subsection{Open problems}

Our study leaves several open problems.

1. Can we deduce assumption $(A)$ just from the regularity of $\Sigma$ and the divergence-free property of $T$ ?

2. If we assume that $\mu$ is supported on just a one-dimensional rectifiable curve, can we obtain similar results? Can we give the best regularity of the curve? Is there an improvement of regularity?

3. Can the support of $\mu$ have a noninteger fractional dimension?

4. To our knowledge, not much is known on equations of the form (3.12) although they seem to be interesting on their own. Natural questions that arise are:

- When the domain $\Omega$ is not thin, does (3.12) have nontrivial solution?

- If $\Omega$ is a ball, is the solution to 3.12 radially symmetric? If this is the case then Proposition 3.1 is still true without the thinness assumption on $\Omega=B_{R}$. 
Recall that for an equation of the form $-\Delta u=f(u)$ in $B_{R}$ with zero Dirichlet boundary condition, if either $f$ has some suitable continuity (see, e.g., [5]) or $f$ has a definite sign (see, e.g., [8]) then $u$ is radially symmetric. In our problem, $f(u)=2 \chi_{\{u>1\}}-u$ is neither continuous nor of a definite sign.

- What is the optimal regularity of the free boundary $\{v=1\}$ ?

\section{Acknowledgments}

I wish to thank my advisor, Professor Sylvia Serfaty, for her insight, encouragement and advice during the course of this work. I would also like to thank Luis Silvestre for helpful discussions. I appreciate the constructive comments on an earlier version of the paper by the anonymous referees.

This research was partially supported by a Vietnam Education Foundation graduate fellowship.

\section{REFERENCES}

1. Ambrosio, L., \& Serfaty, S. A gradient-flow approach for an evolution problem arising in superconductivity. Comm. Pure Appl. Math. 61 (2008), 1495-1539. Zbl pre05358517 MR 2444374

2. AYdi, H. Lines of vortices for solutions of the Ginzburg-Landau equations. J. Math. Pures Appl. 89 (2008), 49-69. Zbl pre05230754 MR 2378089

3. Bethuel, F., Brezis, H., \& HÉlein, F. Ginzburg-Landau Vortices. Progr. Nonlinear Differential Equations Appl. 13, Birkhäuser Boston, Boston, MA (1994). Zbl 0802.35142 MR 1269538

4. Caffarelli, L., Salazar, J., \& Shahgholian, H. Free-boundary regularity for a problem arising in superconductivity. Arch. Ration. Mech. Anal. 171 (2004), 115-128. Zbl 1072.35203 MR 2029533

5. Gidas, B., Ni, W. M., \& Nirenberg, L. Symmetry and related properties via the maximum principle. Comm. Math. Phys. 68 (1979), 209-243. Zbl 0425.35020 MR 0544879

6. Gilbarg, D., \& Hörmander, L. Intermediate Schauder estimates. Arch. Ration. Mech. Anal. 74 (1980), 297-318. Zbl 0454.35022 MR 0588031

7. Gilbarg, D., \& Trudinger, N. S. Elliptic Partial Differential Equations of Second Order. Reprint of the 1998 ed., Classics in Math., Springer, Berlin (2001). Zbl 1042.35002 MR 1814364

8. Lions, P.-L. Two geometrical properties of solutions of semilinear problems. Appl. Anal. 12 (1981), 267-272. Zbl 0445.35043 MR 0653200

9. Majda, A. J., \& Bertozzi, A. L. Vorticity and Incompressible Flow. Cambridge Texts Appl. Math. 27, Cambridge Univ. Press, Cambridge (2002). Zbl 0983.76001 MR 1867882

10. Sandier, E., \& Serfaty, S. Limiting vorticities for the Ginzburg-Landau equations. Duke Math. J. 117 (2003), 403-446. Zbl 1035.82045 MR 1979050

11. SANDier, E., \& Serfaty, S. Vortices in the Magnetic Ginzburg-Landau Model. Progr. Nonlinear Differential Equations Appl. 70, Birkhäuser (2007). Zbl 1112.35002 MR 2279839

12. Serrin, J. A symmetry problem in potential theory. Arch. Ration. Mech. Anal. 43 (1971), 304-318. Zbl 0222.31007 MR 0333220

13. SiRAKOv, B. Symmetry for exterior elliptic problems and two conjectures in potential theory. Ann. Inst. H. Poincaré Anal. Non Linéaire 18 (2001), 135-156. Zbl 0997.35014 MR 1808026

14. Watson, G. N. A Treatise on the Theory of Bessel Functions. Reprint of the second (1944) ed., Cambridge Math. Library, Cambridge Univ. Press, Cambridge (1995). Zbl 0849.33001 MR 1349110 